\title{
Taylor-Couette instability in disk suspensions: Experimental observation and theory
}

\author{
J. J. J. Gillissen $\odot,{ }^{1,}{ }^{*}$ N. Cagney $\odot,,^{2,3}$ T. Lacassagne $\odot,^{3}$ A. Papadopoulou, ${ }^{3}$ \\ S. Balabani $\odot,{ }^{3}$ and H. J. Wilson ${ }^{1}$ \\ ${ }^{1}$ Department of Mathematics, University College London, Gower Street, WC1E 6BT, United Kingdom \\ ${ }^{2}$ School of Engineering and Materials Science, Queen Mary University of London, E1 4NS, United Kingdom \\ ${ }^{3}$ Department of Mechanical Engineering, University College London, WC1E 7JE, United Kingdom
}

(Received 21 January 2020; accepted 28 July 2020; published 19 August 2020)

\begin{abstract}
Using the well-known hydrodynamic theory for dilute suspensions of spheroids, we have previously predicted the destabilization of Taylor-Couette flow due to anisotropic viscous stresses induced by suspended disk-shaped particles [Gillissen and Wilson, Phys. Rev. Fluids 3, 113903 (2018)]. Here we provide experimental evidence for the destabilization mechanism using suspensions of mica flakes. There is good qualitative agreement between the experiment and theory in the mica concentration dependence of the critical speed for instability onset and of the axial wavelength of the corresponding Taylor vortices. Quantitative differences are attributed to hydrodynamic interactions between the disks, which we account for in the theory in an ad hoc fashion using rotary diffusion.
\end{abstract}

DOI: 10.1103/PhysRevFluids.5.083302

\section{INTRODUCTION}

Taylor-Couette flow (TCF) is the flow in the gap between two counter-rotating cylinders. When the outer cylinder is held fixed and the rotation speed of the inner cylinder exceeds a threshold value, the circular Taylor-Couette base flow destabilizes, which is accompanied by the emergence of so-called Taylor vortices [1]. For Newtonian fluids, the onset of instability corresponds to the Taylor number:

$$
\mathrm{Ta}=\frac{\rho \Omega \sqrt{\Delta R^{3} R_{1}}}{\eta_{s}},
$$

exceeding a critical value $\mathrm{Ta}_{c}$ that depends on the cylinder radius ratio $R_{1} / R_{2}$. Here $\eta_{s}$ is the fluid viscosity, $\Omega$ is the angular velocity of the inner cylinder, $\Delta R=R_{2}-R_{1}$ is the gap width between the cylinders, and $R_{1}$ and $R_{2}$ are the radii of the inner and of the outer cylinder, respectively.

In non-Newtonian fluids the behavior is different, and two types of non-Newtonian TCF instabilities have been observed. The first type is driven by centrifugal forces, similar to the Newtonian instability, described above. In this case the non-Newtonian rheology only alters the details of the instability, i.e., the onset speed, the shape, and the dynamics of the Taylor vortices, while the driving mechanism (centrifugal force) remains the same. Examples of this type of instability include fluids with a shear thinning rheology [2,3], such as dilute solutions of rodlike polymers, e.g., carboxymethyl cellulose, polyacrylic acid, and xanthan [4-6]. Other examples include suspensions of non-Brownian ellipsoids [7,8] and dense suspensions of spheres [9-11], where in the latter case, the non-Newtonian effects arise from an anisotropic microstructure [12-15]

*jurriaangillissen@gmail.com 

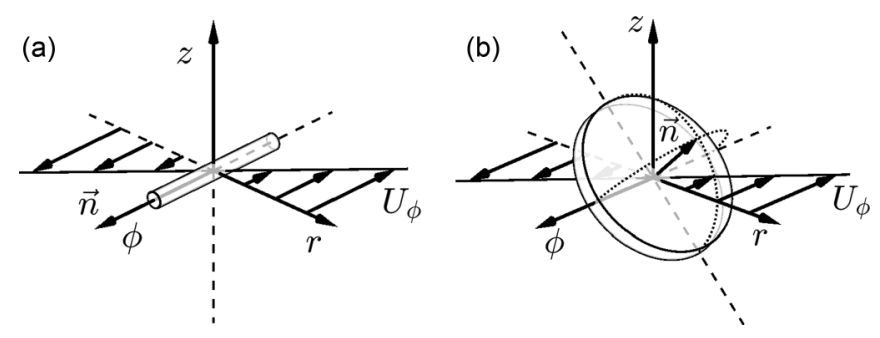

FIG. 1. (a) A rod with its major axis $\boldsymbol{n}$ in the azimuthal direction $\phi$ does not rotate when subjected to an azimuthal vorticity perturbation. (b) A disk, on the other hand, tilts its normal $\boldsymbol{n}$ away from the radial direction $r$ towards the axial direction $z$. The mean flow field $U_{\phi}(r)$ is drawn relative to the motion of the particles.

as well as from a heterogeneous solid concentration $[9,16]$. Dilute suspensions of spherical particles (with volume fraction $c \lesssim 0.05$ ) roughly behave as Newtonian fluids with an elevated viscosity, which may induce a very small destabilizing effect due to particle inertia [16].

The second type of non-Newtonian TCF instability is driven by viscoelastic stresses and persists even in the absence of centrifugal forces. This so-called "elastic instability" has been observed in polymer solutions [17-19] and in micelle solutions [20]. The elastic instability is well understood and reproduced by numerical simulation using constitutive equations of viscoelastic fluids [18,21]. Other instabilities, related to non-Newtonian rheology, include shear banding and vorticity banding [22], but these are not specific for the TCF geometry.

In this work we report on the modification of the centrifugal TCF instability due to anisotropic viscous stresses generated by suspended disk-shaped particles. In a previous work we theoretically predicted that, for suspensions of perfectly aligned disks, i.e., disks with an infinite aspect ratio and zero rotary diffusivity $D_{r}$, the instability persists even when the centrifugal force becomes vanishingly small [8]. Zero rotary diffusion corresponds to an infinite rotary Péclet number:

$$
\mathrm{Pe}=\frac{\dot{\gamma}}{D_{r}}
$$

where $\dot{\gamma}$ is the shear rate, $D_{r} \sim k_{B} T /\left(\eta_{s} l^{3}\right)$, and $l$ is the major axis of the disks. Under these idealized conditions, the critical Taylor number [Eq. (1)] required for instability onset was equal to zero. As $\mathrm{Ta} \rightarrow 0$, the instability growth rate $\lambda$ decreases as $\lambda \sim v \Delta R^{-2} \mathrm{Ta}^{2}$ (Fig. 4(b) in Ref. [8]), i.e., a nonzero growth rate requires a nonzero centrifugal force. In Ref. [8], it was furthermore predicted that nonidealized conditions, i.e., a finite rotary diffusivity and a finite aspect ratio, have an adverse effect on the instability, resulting in a nonzero critical Taylor number.

A related but not entirely similar destabilization mechanism has been observed in suspensions of disk-shaped clay particles [23]. These clay suspensions also generate elastic stresses and are strongly shear thinning due to rotary Brownian diffusion and electrostatic interparticle forces [24]. Shear thinning destabilizes TCF, even in the absence of anisotropic viscous stresses [2].

In Fig. 1 we explain the destabilizing effect of suspended disks by contrasting it to the negligible effect of suspended rods. Figure 1(a) illustrates a rod in the Taylor-Couette base flow which has a strain rate $s_{\phi r}$. The flow, gradient, and vorticity directions are $\phi, r$, and $z$, respectively. In the limit of an infinite aspect ratio and an infinite Péclet number [Eq. (2)], the rod major axis $\boldsymbol{n}$ points in the $\phi$ direction, where it generates no hydrodynamics stress. A Taylor vortex perturbation corresponds to azimuthal fluid vorticity $\omega_{\phi}^{\prime}$, i.e., to fluid rotation around $\boldsymbol{n}$. Consequently, $\boldsymbol{n}$ remains fixed and the rod generates no additional stress.

For a disk, the situation is sketched in Fig. 1(b). In the base flow, the disk normal $\boldsymbol{n}$ points in the $r$ direction. A Taylor vortex perturbation $\omega_{\phi}^{\prime}$ rotates $\boldsymbol{n}$ away from the $r$ axis and towards the $z$ axis. The perturbation of the disk normal in the $z$ direction $n_{z}^{\prime}$ generates a stress perturbation 

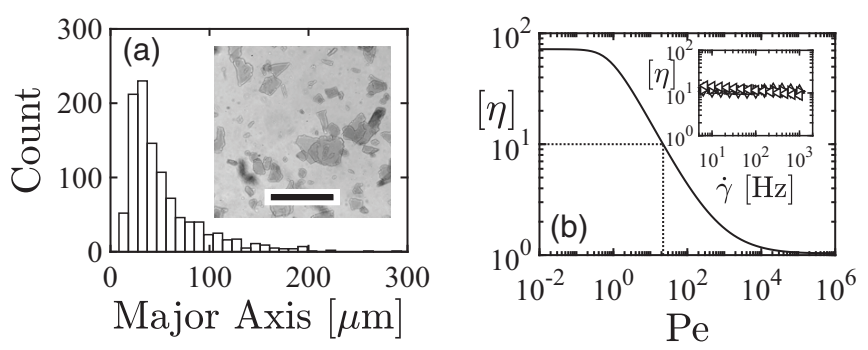

FIG. 2. (a) Mica size distribution, obtained from micrographs, as shown in the inset, which has a $200-\mu \mathrm{m}$ scale bar. (b) The theoretical, scaled disk viscosity $[\eta]$ as a function of the rotary Péclet number Pe in dilute suspensions of disks with an aspect ratio of $r_{a}^{-1}=10^{2}$. The dotted lines indicate $\mathrm{Pe} \approx 22$, which corresponds to the experimentally measured $[\eta] \approx 10$. The inset shows the experimentally measured $[\eta]$ in mica suspensions as a function of the shear rate $\dot{\gamma}$ for mica volume fractions of $c=5 \times 10^{-3}$ (downward triangle), $c=10^{-2}$ (upward triangle), $c=2 \times 10^{-2}$ (rightward triangle), and $c=5 \times 10^{-2}$ (leftward triangle).

$\sigma_{\phi z}^{\prime} \sim s_{\phi r} n_{r} n_{z}^{\prime}$ [see Eq. (9) below]. This stress perturbation has an amplifying feedback on the Taylor vortex perturbation $\omega_{\phi}^{\prime}$ via $\partial_{t} u_{\phi}^{\prime} \sim \partial_{z} \sigma_{\phi z}^{\prime}$ and $\partial_{t} u_{r}^{\prime} \sim(U / R) u_{\phi}^{\prime}$ [see Eq. (8) below] and $\omega_{\phi}^{\prime} \sim \partial_{z} u_{r}^{\prime}$.

\section{EXPERIMENTS}

In this work we provide experimental evidence for the destabilizing effect of TCF due to suspended, non-Brownian, and (nearly) nonadhesive disks. To this end, we use suspensions of mica flakes (Cornelius Ltd.) with a thickness of $d \approx 1 \mu \mathrm{m}$ and a mass density of $2.93 \mathrm{~g} \mathrm{~cm}^{-3}$. Figure 2(a) shows the distribution of the major particle axis $l$ which is obtained from 20 micrographs, as shown in the inset of Fig. 2(a). In addition to inducing hydrodynamic instability, the mica flakes also serve to visualize the flow structures. The reflectivity of the suspension depends on the relative orientation of the incoming and the outgoing light w.r.t to the orientation of the flakes, which in turn is governed by the various components of the fluid velocity gradient tensor [see Eq. (10) below].

We examine one Newtonian fluid, i.e., with a very low flake volume fraction $c=10^{-4}$, and five suspensions with flake volume fractions ranging between $c=10^{-3}$ and $c=5 \times 10^{-2}$. The suspending medium is a mixture of glycerol (volume fraction $G$ ), distilled water (volume fraction $\mathrm{W}$ ), and aqueous food dye to aid flow visualization (volume fraction 0.02). For $c \leqslant 10^{-2}$ and $c \geqslant$ $2 \times 10^{-2}$ we used $(\mathrm{G}, \mathrm{W})=(0.71,0.27)$ and $(0.9,0.08)$, respectively, which correspond to a density and a viscosity of $\left(\rho\left[\mathrm{g} \mathrm{cm}^{-3}\right], \eta_{s}[\mathrm{~Pa} \mathrm{~s}]\right)$ of $(1.18,0.036)$ and $(1.24,0.3)$, respectively. The more viscous liquid was used to suppress sedimentation effects at the higher mica concentrations.

The steady shear viscosity $\eta_{\text {eff }}$ of the suspensions is measured using a rotational rheometer (TA Instruments) equipped with a cone-and-plate geometry. The inset of Fig. 2(b) shows the measured viscosity induced by the disks divided by the disk volume fraction, which we refer to as the scaled disk viscosity:

$$
[\eta]=\frac{\eta_{\mathrm{eff}}-\eta_{s}}{c \eta_{s}}
$$

as a function of the shear rate $\dot{\gamma}$ for the various suspensions. The shear rate range $5 \leqslant \dot{\gamma} \leqslant 10^{3} \mathrm{~s}^{-1}$ would correspond to a Taylor number [Eq. (1)] range in the TCF setup of approximately $3 \leqslant \mathrm{Ta} \leqslant$ $6 \times 10^{2}$. For $c \leqslant 2 \times 10^{-2}$, the measured [ $\eta$ ] collapse, i.e., $[\eta]$, is independent of $c$ and $\dot{\gamma}$, causing overlapping (and therefore invisible) markers in the inset of Fig. 2(b). For $c=5 \times 10^{-2}$, there is slight shear thinning, i.e., the exponent $\delta$ in $[\eta] \sim \dot{\gamma}^{-\delta}$ is around $\delta \approx 0.02$. This exponent is much smaller than in suspensions of more adhesive platelets, such as graphene [25] and clay [24], whose exponents have been observed in the range $0.5<\delta<1$. The (near) rate independence of our mica 

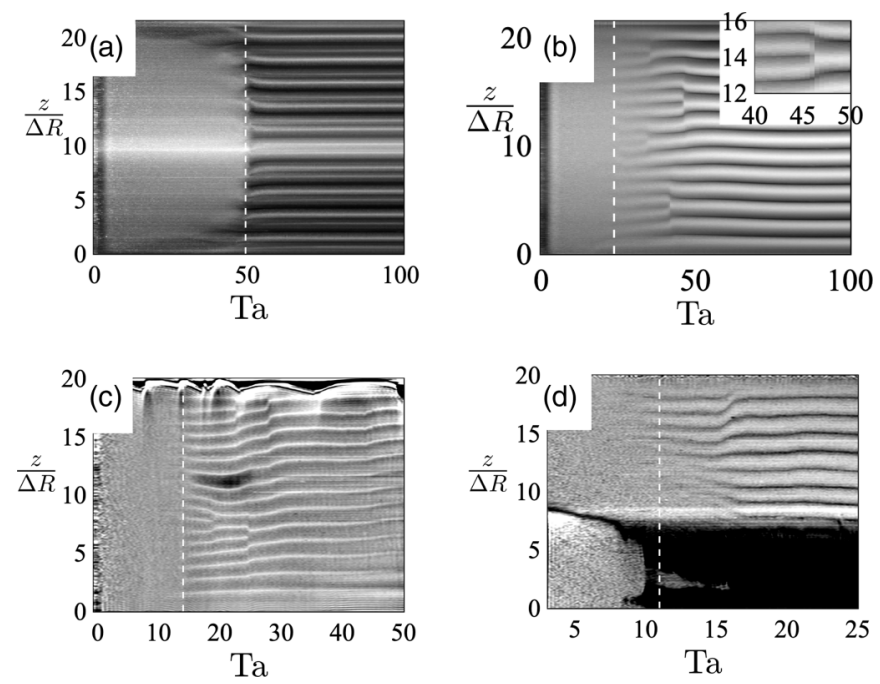

FIG. 3. Light intensity maps $I\left(z\right.$, Ta) using $\left[c, d \Omega^{*} / d t^{*}\right]$ equal to $\left[10^{-4}, 0.7\right]$ (a), $\left[10^{-2}, 0.7\right]$ (b), $[2 \times$ $\left.10^{-2}, 0.9\right]$ (c), and $\left[5 \times 10^{-2}, 0.6\right](\mathrm{d})$. The maps show the onset of instability as the appearance of the banded structures above a critical speed, indicated by the dashed white lines. The inset of (b) shows a closeup of a vortex merger event.

suspensions, i.e., small $\delta$, confirms the (near) absence of adhesion forces and corresponding elastic behavior.

The cylinders in the TCF setup have length $L=155 \mathrm{~mm}$ and radii $R_{1}=21.66 \mathrm{~mm}$ and $R_{2}=$ $27.92 \mathrm{~mm}$, which correspond to a radius ratio of $R_{1} / R_{2}=0.77$ and an aspect ratio of $L / \Delta R=$ 21.56. The flow cell is enclosed within a rectangular chamber in which water is recirculated to keep the fluid temperature in the flow cell at $20^{\circ} \mathrm{C} \pm 0.1^{\circ} \mathrm{C}$ [6]. The inner cylinder is accelerated from rest with a constant acceleration rate $d \Omega / d t$. The flow cell is illuminated using a white light-emitting diode (SugarCUBE, Edmund Optics). We use a CMOS camera (Phantom Miro 340) to image a strip of the flow cell with $2224 \times 16$ pixels in the $z$ and $\phi$ directions and a frame rate of $60 \mathrm{~s}^{-1}$. Each image is averaged over the 16 pixels in the $\phi$ direction into a $z$ profile with 2224 pixels. The resulting profiles are combined into a matrix which is referred to as the light intensity map $I(z, \mathrm{Ta})$. This map is a function of the height $z$ and of the effective Taylor number Ta:

$$
\mathrm{Ta}=\frac{\rho \Omega \sqrt{\Delta R^{3} R_{1}}}{\eta_{\text {eff }}} .
$$

Here $\eta_{\text {eff }}$ is the measured, effective viscosity; see inset of Fig. 2(b).

In Fig. 3 we show $I(z, \mathrm{Ta})$ for mica concentrations ranging from $c=10^{-4}$ to $c=5 \times 10^{-2}$. The figure shows that, above a critical Taylor number $\mathrm{Ta}_{c}$, the circular base flow transitions into a vortical flow, indicated by the appearance of bright and dark bands in $I(z$, Ta). For the Newtonian suspension, with negligible mica concentration $c=10^{-4}$ [Fig. 3(a)], the instability starts at both ends in the form of Ekman vortices. Since these end effects are not associated with the Taylor vortices, we disregard the end regions in the subsequent analysis. For $c=2 \times 10^{-2}$ [Fig. 3(c)] the visualization near the top was complicated (reflected by the undulations) due to a leak in the external cooling circuit with the cooling water level dropping off and being readjusted as the experiment was running. For $c=5 \times 10^{-2}$ [Fig. 3(d)], sedimentation effects are manifested by the dark region in the lower half. These regions are disregarded from the subsequent analysis. Nevertheless, axial concentration variations are expected to be weak, since the gravity force $\Delta \rho g d l^{2}$ is four orders of magnitude smaller than the hydrodynamic force $\dot{\gamma} \eta_{\text {eff }} l^{2}$, where $g=9.81 \mathrm{~m} \mathrm{~s}^{-2}$ is the gravitational 
acceleration and $\Delta \rho=2.34 \mathrm{~g} \mathrm{~cm}^{-3}$ is the density difference between the mica and the suspending medium.

In Figs. 3(b)-3(d), faint ridges appear for $\mathrm{Ta}>\mathrm{Ta}_{c}$ which gradually become more distinct as $\mathrm{Ta}$ is increased further. This indicates that at $\mathrm{Ta}=\mathrm{Ta}_{c}$ the Taylor vortex strength is relatively weak and it grows for $\mathrm{Ta}>\mathrm{Ta}_{c}$. This gradual development of the Taylor vortex strength is not observed in the Newtonian system [Fig. 3(a)] nor in similar measurements of solutions of flexible or rodlike polymers; see, e.g., Ref. [6].

In Ref. [8] we predicted that $\mathrm{Ta}_{c}=0$ under the hypothetical conditions of noninteracting disks with an infinite aspect ratio. The observation of a nonzero $\mathrm{Ta}_{c}$ in Figs. 3(b)-3(d) reflects non-negligible hydrodynamic interactions in these mica suspensions as well as a finite aspect ratio.

The light intensity map for $c=10^{-2}$ in Fig. 3(b) shows another interesting feature; as Ta is increased, the number of vortices (indicated by the number of bright and dark bands) abruptly decreases at several points. These events correspond to the merger of two adjacent vortices. A closeup of such a vortex merger event is provided in the inset of Fig. 3(b). This phenomenon has also been observed in solutions of polymers $[6,26]$. It is noted that these sudden changes are likely due to the finite $L / \Delta R$ of the TCF setup, while for $L / \Delta R \rightarrow \infty$ these changes are expected to be continuous.

The onset of instability corresponds to critical values, $k_{c}$ and $\mathrm{Ta}_{c}$, of the axial vortical wave number $k$ and of the effective Taylor number [Eq. (4)]. To determine $k_{c}$ and $\mathrm{Ta}_{c}$, the $I(z$, Ta) (Fig. 3) are first filtered over Ta with a filter width of $\Delta \mathrm{Ta} \approx 1$. This is to improve the statistical significance of the variations of $I(z, \mathrm{Ta})$ with Ta. Then for each value of Ta we compute the Fourier transformed light intensity map:

$$
\hat{I}(k, \mathrm{Ta})=\int \exp (i z k) I(z, \mathrm{Ta}) d z
$$

For each Ta we determine the maximum $\hat{I}_{m}$ of $\hat{I}(k$, Ta $)$ as a function of $k$, excluding the $k=0$ mode. The maximum $\hat{I}_{m}$ occurs at wave number $k_{m}$.

Figure 4(a) shows $\hat{I}_{m}$ and $k_{m}$ as functions of Ta for the Newtonian system [Fig. 3(a)] using $c=$ $10^{-4}$ and a nondimensional ramp-up speed of $d \Omega^{*} / d t^{*} \approx 0.7$ [27]:

$$
\frac{d \Omega^{*}}{d t^{*}}=\frac{\rho^{2} R_{1} \Delta R^{3}}{\eta_{\mathrm{eff}}^{2}} \frac{d \Omega}{d t} .
$$

As Ta passes the critical value $\mathrm{Ta}_{c}$, indicated by the vertical dotted line, $\hat{I}_{m}$ starts growing, which corresponds to the onset of Taylor vortices. For this Newtonian case, we find a critical Taylor number of $\mathrm{Ta}_{c} \approx 46$, which is close to the theoretical value of $\mathrm{Ta}_{c} \approx 48$ [28]. The critical wave number $k_{c}$ is determined as $k_{m}$ at $\mathrm{Ta}=\mathrm{Ta}_{c}$, giving $k_{c} \Delta R / \pi \approx 0.92$, which is close to the theoretical value of $k_{c} \Delta R / \pi \approx 1.0$. The agreement between these experimental results and the literature values confirms that the nondimensional ramp-up speed of $d \Omega^{*} / d t^{*} \approx 0.7$ is sufficiently slow to ensure that these Newtonian results are not affected by the finite acceleration rate.

Figures 4(b)-4(d) show $\hat{I}_{m}$ and $k_{m}$ as functions of Ta for $c=10^{-2}$ and for three values for $d \Omega^{*} / d t^{*}$. It can be seen that for $d \Omega^{*} / d t^{*} \approx 0.1$ and 0.7 the critical values $\mathrm{Ta}_{c}$ and $k_{c}$ are close to one another, i.e., $\mathrm{Ta}_{c} \approx 19.7$ and 21.6 and $k_{c} \approx 1.55$ and 1.51 . For the larger $d \Omega^{*} / d t^{*} \approx 7$, on the other hand, the critical values deviate somewhat, i.e., $\mathrm{Ta}_{c} \approx 26.0$ and $k_{c} \approx 1.38$. These results show that for $c=10^{-2}, d \Omega^{*} / d t^{*} \approx 0.7$ is sufficiently slow to obtain critical values that are (nearly) independent of $d \Omega^{*} / d t^{*}$. We use a similar acceleration rate of $d \Omega^{*} / d t^{*} \approx 0.9$ for $c=2 \times 10^{-2}$ [Fig. 4(e)] and $d \Omega^{*} / d t^{*} \approx 0.6$ for $c=5 \times 10^{-2}$ [Fig. 4(f)]. It is finally noted that the vortical wavelength $k_{m}$ in Figs. 4(b)-4(f) shows sudden variations which are associated with the vortex merger events, as shown in Fig. 3(b). 

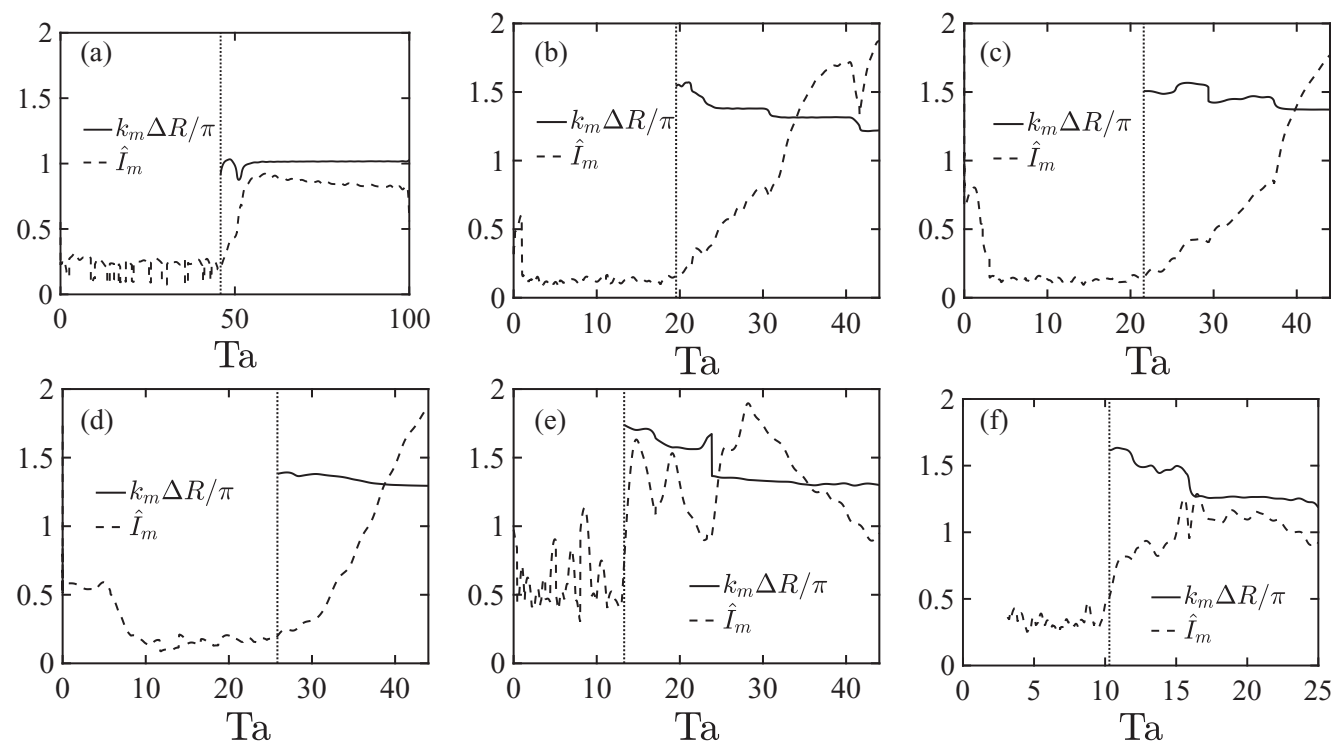

FIG. 4. The maximum $\hat{I}_{m}$ (arbitrary units) of the Fourier transformed light intensity map [Eq. (5)] and the corresponding wave number $k_{m}$ as functions of Ta for $\left[c, d \Omega^{*} / d t^{*}\right]$ equal to $\left[10^{-4}, 0.7\right]$ (a), [10 $\left[0^{-2}, 0.1\right]$ (b), $\left[10^{-2}, 0.7\right](\mathrm{c}),\left[10^{-2}, 7\right](\mathrm{d}),\left[2 \times 10^{-2}, 0.9\right](\mathrm{e})$, and $\left[5 \times 10^{-2}, 0.6\right]$ (f). The critical Taylor number $\mathrm{Ta}_{c}$ is indicated with the vertical dotted lines which correspond to the growth onset of $\hat{I}_{m}$.

\section{THEORY}

We now compare the experimental results to the theoretical model of Ref. [8]. The model is based on the well-known constitutive equations for dilute suspensions of spheroids which are given by the continuity equation

$$
\nabla \cdot \boldsymbol{u}=0
$$

and the momentum equation

$$
\rho \partial_{t} \boldsymbol{u}=\nabla \cdot\left[-\rho \boldsymbol{u} \boldsymbol{u}-p \boldsymbol{\delta}+\eta_{s}\left(\boldsymbol{\nabla} \boldsymbol{u}+\nabla \boldsymbol{u}^{T}\right)+\boldsymbol{\sigma}\right] .
$$

In Eq. (8), the spheroid stress $\sigma$ is given by [29]

$$
\frac{\boldsymbol{\sigma}}{\eta_{s}}=2 \alpha_{1} \boldsymbol{s}+2 \alpha_{2} \boldsymbol{s}: \boldsymbol{a} \boldsymbol{a}+\alpha_{3}(\boldsymbol{s} \cdot \boldsymbol{a}+\boldsymbol{a} \cdot \boldsymbol{s})+\alpha_{4} D_{r}\left(\boldsymbol{a}-\frac{1}{3} \boldsymbol{\delta}\right),
$$

which depends on the microstructure $\boldsymbol{a}=\langle\boldsymbol{n} \boldsymbol{n}\rangle$. Here $\boldsymbol{n}$ is the unit vector along the spheroid polar axis (Fig. 1) and $\langle\cdots\rangle$ denotes the average over a statistical ensemble of spheroids. The microstructure tensor $\boldsymbol{a}$ evolves as

$$
\partial_{t} \boldsymbol{a}=-\boldsymbol{u} \cdot \nabla \boldsymbol{a}+\nabla \boldsymbol{u}^{T} \cdot \boldsymbol{a}+\boldsymbol{a} \cdot \nabla \boldsymbol{u}+(B-1)(\boldsymbol{s} \cdot \boldsymbol{a}+\boldsymbol{a} \cdot \boldsymbol{s})-2 B \boldsymbol{s}: \boldsymbol{a} \boldsymbol{a}-D_{r}\left(\boldsymbol{a}-\frac{1}{3} \boldsymbol{\delta}\right) .
$$

Here $\boldsymbol{u}$ is the velocity, $\rho$ is the suspension mass density, $p$ is the pressure, $\boldsymbol{s}=\frac{1}{2}\left(\boldsymbol{\nabla} \boldsymbol{u}+\boldsymbol{\nabla} \boldsymbol{u}^{T}\right)$ is the rate of strain tensor, $D_{r}$ is the rotary diffusivity, which is added to mimic the effects of hydrodynamic interactions between the non-Brownian disks, $c$ is the spheroid volume fraction, $r_{a}=a / b$ is the aspect ratio, $a$ is the polar radius, $b$ is the equatorial radius, $\alpha_{i}$ are material constants that depend on $c$ and $r_{a}$, and $B=\left(r_{a}^{2}-1\right) /\left(r_{a}^{2}+1\right)$. The cases $r_{a}<1, r_{a}=1$, and $r_{a}>1$ correspond to oblate spheroids (disks), spheres, and prolate spheroids (rods), respectively.

In order to estimate the effective aspect ratio that corresponds to the size distribution in Fig. 2(a), we use that the disk stress $\sigma$ scales with the disk major axis cubed [29]. Therefore the relevant 


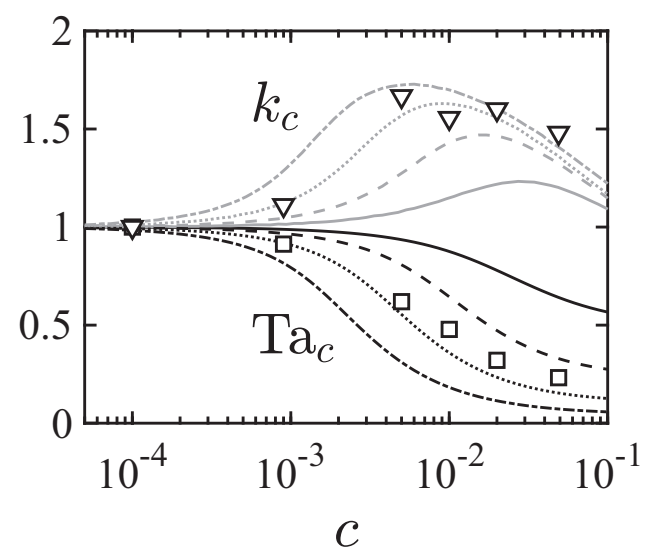

FIG. 5. The critical Taylor number $\mathrm{Ta}_{c}$ (black lines and squares) and the critical vortical wave number $k_{c}$ (gray lines and triangles), normalized by their Newtonian values, plotted vs the disk concentration $c$. Comparison between experimental data (markers) and theory (lines). The theory uses an aspect ratio of $r_{a}^{-1}=10^{2}$ and a rotary Péclet number of $\mathrm{Pe}=1 \times 10^{2}$ (solid lines), $\mathrm{Pe}=1 \times 10^{3}$ (dashed lines), $\mathrm{Pe}=1 \times 10^{4}$ (dotted lines), and $\mathrm{Pe}=1 \times 10^{5}$ (dash-dotted lines).

particle dimension is the cube root of the third moment of this distribution $l_{\mathrm{eff}}=\left\langle l^{3}\right\rangle^{1 / 3} \sim 10^{2} \mu \mathrm{m}$, giving an aspect ratio of $r_{a}^{-1}=l_{\text {eff }} / d \sim 10^{2}$.

In the theory [Eqs. (7)-(10)] hydrodynamic interactions are not taken into account rigorously. The number of these interactions per particle is proportional to the volume fraction of the disk-circumscribing spheres $\sim c r_{a}^{-1}$. In the present work we consider mica suspensions with concentrations up to $c r_{a}^{-1} \sim 5$, for which hydrodynamic interactions are expected to be important. We model these interactions as rotary diffusion $\left(D_{r}\right.$ terms) in Eqs. (9) and (10). Theoretical and experimental studies have shown that rotary diffusion is an adequate model for hydrodynamic interactions between rods [30-34]. For disks, on the other hand, there are no equivalent studies, and at present it is not clear if interactions between disks can adequately be modeled by rotary diffusion. We shed some light on this issue below by comparing the theoretical model [Eqs. (7)-(10)] to experimental data, for both steady shear flow and for the TCF instability.

First we show in Fig. 2(b) the theoretical [Eqs. (7)-(10)] scaled disk viscosity $[\eta]$ [Eq. (3)] in the steady shear flow of a suspension of oblate spheroids with an aspect ratio of $r_{a}^{-1}=10^{2}$ as a function of the rotary Péclet number [Eq. (2)]. The theoretical viscosity in Fig. 2(b) decreases as a function of Pe. For Pe $\approx 22$ the model matches the experimental data $[\eta] \approx 10$ [inset of Fig. 2(b)]. We reemphasize that the mica flakes are non-Brownian and that rotary diffusion is used as a model for the effects of hydrodynamic interactions between the disks. We further note that the (near) shear rate $\dot{\gamma}$ invariance of the measured $[\eta]$ [inset in Fig. 2(b)] indicates a constant rotary Péclet number [Eq. (2)], i.e., $D_{r} \sim \dot{\gamma}$.

We present linear stability analysis of the cylindrical coordinate version of Eqs. (7)-(10) with respect to axisymmetric perturbations $u^{\prime}(r) \exp (\dot{\mathrm{i}} k z) \exp (\lambda t)$ where $k$ is the axial wave number and $\lambda$ is the growth rate. The axisymmetry of the instability modes is experimentally observed in Fig. 3 . Details of the stability analysis are given in Ref. [8]. Briefly, we discretize Eqs. (7)-(10) using 30 Chebyshev collocation points. After computing the base state, we compute $\lambda$ by numerically solving the corresponding generalized eigenvalue problem. For all cases shown, $\lambda$ is found to be real-valued, i.e., nonoscillatory, in agreement with the experimental observations in Fig. 3.

To match the experimental system, we use a radius ratio of $R_{1} / R_{2}=0.77$, a disk aspect ratio of $r_{a}^{-1}=10^{2}$, and we vary the disk concentration between $c=10^{-4}$ and $c=10^{-1}$ and the rotary Péclet number between $\mathrm{Pe}=10^{2}$ and $\mathrm{Pe}=10^{5}$. For each $c$ and Pe we vary the wave number $k$ of the perturbation, and for each $k$ we vary the rotation speed $\Omega$. We thereby find the critical wave 

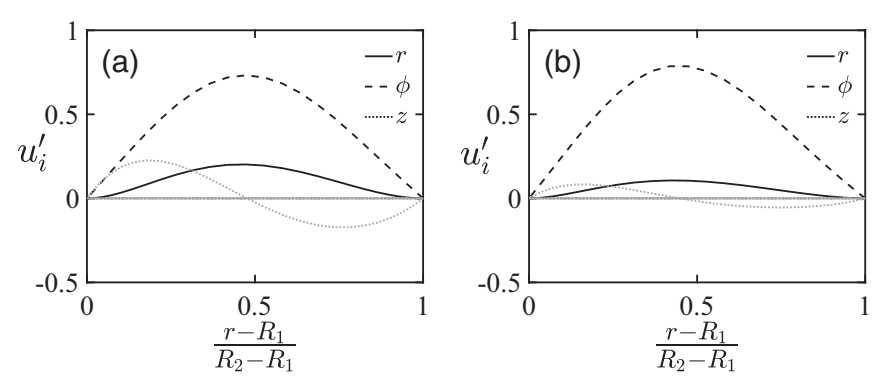

FIG. 6. Real part (black) and imaginary part (gray) of the theoretically computed Taylor vortex in a Newtonian system (a) and in a disk suspension (b) using an aspect ratio of $r_{a}^{-1}=10^{2}$, a rotary Péclet number of $\mathrm{Pe}=3 \times 10^{3}$, and a concentration of $c=10^{-2}$.

number $k_{c}$ and the critical Taylor number $\mathrm{Ta}_{c}$ that mark the transition between positive and negative $\lambda$, i.e., the onset of instability.

Figure 5 shows good qualitative agreement between the computed and measured $\mathrm{Ta}_{c}$ and $k_{c}$ as functions of $c$. The experimentally measured $k_{c}$ suggest a slight discontinuity between $c=10^{-2}$ and $c=2 \times 10^{-2}$ which is likely due to the change in the suspending medium (see Sec. II) and the corresponding changes in sedimentation and interparticle adhesion. These effects are expected to be weak, however, since the measured $\mathrm{Ta}_{c}$ (Fig. 5) does not suggest a discontinuity. The experimental data for $\mathrm{Ta}_{c}$ agree well with the numerical results for $10^{3} \lesssim \mathrm{Pe} \lesssim 10^{4}$. This range is beyond the value of $\mathrm{Pe} \approx 22$, which was required to match the constitutive model to the experimental data for steady shear flow [Fig. 2(b)]. This discrepancy highlights that rotary diffusion is not an accurate model for hydrodynamic interactions between disks. Indeed, hydrodynamic interactions between disks are more complicated than a mere randomizing effect. In contrast to rotary diffusion, hydrodynamic repulsion may suppress rotational freedom [35]. Nevertheless, there is good qualitative agreement between the theory and the experimental data in both $\mathrm{Ta}_{c}$ and $k_{c}$ as functions of $c$. This agreement supports our theoretical finding [8] that Taylor-Couette flow can be destabilized by anisotropic viscous stresses due to suspended disk-shaped particles. The agreement also indicates that sedimentation, although visible in Fig. 3(d), does not have a dramatic effect, i.e., the mica concentration in the upper half of Fig. 3(d) is expected to be close to the volume-averaged value $c$.

Figure 6 shows the theoretically computed velocity profiles of the Taylor vortices with $c=0$ and $\mathrm{Ta}=\mathrm{Ta}_{c} \approx 48$ and with $c=10^{-2}$ and $\mathrm{Ta}=\mathrm{Ta}_{c} \approx 24$. Compared to the Newtonian Taylor vortex [Fig. 6(a)], the Taylor vortex in the disk suspension [Fig. 6(b)] has a suppressed cross-stream velocity. These results agree qualitatively with the light intensity maps in Fig. 3, showing that the Newtonian Taylor vortex has a relatively large intensity immediately at $\mathrm{Ta}=\mathrm{Ta}_{c}$ which stays roughly constant for $\mathrm{Ta}>\mathrm{Ta}_{c}$, whereas the non-Newtonian Taylor vortices have a relatively small intensity at $\mathrm{Ta}=\mathrm{Ta}_{c}$ which increases for $\mathrm{Ta}>\mathrm{Ta}_{c}$.

\section{CONCLUSIONS}

We have previously theoretically predicted that Taylor-Couette flow can be destabilized by anisotropic viscous stresses induced by suspended disk-shaped particles [8]. These particles redirect the transfer of azimuthal momentum from the radial to the axial direction. The theory was based on the well-known constitutive equations of suspensions of noninteracting spheroids.

In this work we have provided experimental evidence for this destabilization mechanism using suspensions of mica flakes. In order to match the theory to the experimental data, we have added a rotary diffusion term to the constitutive equations which models the hydrodynamic interactions between the disks. With this modification, there is good qualitative agreement between theory and 
experiment in the concentration dependence of the critical speed for instability onset and of the Taylor vortex size. We believe that quantitative differences between the theory and the experiments are mainly due to inaccurate modeling of hydrodynamic interactions as rotary diffusion, and to a lesser extent due to (weak) adhesion forces and sedimentation effects.

This destabilization mechanism has a range of potential industrial applications, e.g., to enhance mixing in chemical reactors, to enhance heat transfer in drilling equipment, or to better control the exfoliation of graphite into graphene [36].

\section{ACKNOWLEDGMENTS}

This work is supported by the Engineering and Physical Sciences Research Council of the United Kingdom through Grant No. EP/N024915/1.

[1] G. I. Taylor, Stability of a viscous liquid contained between two rotating cylinders, Philos. Trans. R. Soc. London, Ser. A 223, 289 (1923).

[2] T. J. Lockett, S. M. Richardson, and W. J. Worraker, The stability of inelastic non-Newtonian fluids in Couette flow between concentric cylinders: A finite-element study, J. Non-Newtonian Fluid Mech. 43, 165 (1992).

[3] B. Alibenyahia, C. Lemaitre, C. Nouar, and N. Ait-Messaoudene, Revisiting the stability of circular Couette flow of shear-thinning fluids, J. Non-Newtonian Fluid Mech. 183, 37 (2012).

[4] V. Sinevic, R. Kuboi, and A. W. Nienow, Power numbers, Taylor numbers and Taylor vortices in viscous Newtonian and non-Newtonian fluids, Chem. Eng. Sci. 41, 2915 (1986).

[5] M.-K. Yi and C. Kim, Experimental studies on the Taylor instability of dilute polymer solutions, J. NonNewtonian Fluid Mech. 72, 113 (1997).

[6] N. Cagney and S. Balabani, Taylor-Couette flow of shear-thinning fluids, Phys. Fluids 31, 053102 (2019).

[7] V. K. Gupta, R. Sureshkumar, B. Khomami, and J. Azaiez, Centrifugal instability of semidilute nonBrownian fiber suspensions, Phys. Fluids 14, 1958 (2002).

[8] J. J. J. Gillissen and H. J. Wilson, Taylor Couette instability in disk suspensions, Phys. Rev. Fluids 3, 113903 (2018).

[9] M. V. Majji and J. F. Morris, Inertial migration of particles in Taylor-Couette flows, Phys. Fluids 30, 033303 (2018).

[10] P. Ramesh, S. Bharadwaj, and M. Alam, Suspension Taylor-Couette flow: Co-existence of stationary and traveling waves, and the characteristics of Taylor vortices and spirals, J. Fluid Mech. 870, 901 (2019).

[11] J. J. J. Gillissen and H. J. Wilson, Taylor-Couette instability in sphere suspensions, Phys. Rev. Fluids 4, 043301 (2019).

[12] J. J. J. Gillissen and H. J. Wilson, Modeling sphere suspension microstructure and stress, Phys. Rev. E 98, 033119 (2018).

[13] J. J. J. Gillissen and H. J. Wilson, Effect of normal contact forces on the stress in shear rate invariant particle suspensions, Phys. Rev. Fluids 4, 013301 (2019).

[14] J. J. J. Gillissen, C. Ness, J. D. Peterson, H. J. Wilson, and M. E. Cates, Constitutive Model for TimeDependent Flows of Shear-Thickening Suspensions, Phys. Rev. Lett. 123, 214504 (2019).

[15] J. J. J. Gillissen, C. Ness, J. D. Peterson, H. J. Wilson, and M. E. Cates, Constitutive model for shearthickening suspensions: Predictions for steady shear with superposed transverse oscillations, J. Rheol. 64, 353 (2020).

[16] M. E. Ali, D. Mitra, J. A. Schwille, and R. M. Lueptow, Hydrodynamic stability of a suspension in cylindrical Couette flow, Phys. Fluids 14, 1236 (2002).

[17] R. H. Thomas and K. Walters, The stability of elastico-viscous flow between rotating cylinders, Part 1, J. Fluid Mech. 18, 33 (1964).

[18] R. G. Larson, E. S. G. Shaqfeh, and S. J. Muller, A purely elastic instability in Taylor-Couette flow, J. Fluid Mech. 218, 573 (1990). 
[19] Alexander Groisman and Victor Steinberg, Couette-Taylor Flow in a Dilute Polymer Solution, Phys. Rev. Lett. 77, 1480 (1996).

[20] M. A. Fardin, D. Lopez, J. Croso, G. Grégoire, O. Cardoso, G. H. McKinley, and S. Lerouge, Elastic Turbulence in Shear Banding Wormlike Micelles, Phys. Rev. Lett. 104, 178303 (2010).

[21] N. Liu and B. Khomami, Elastically induced turbulence in Taylor-Couette flow: Direct numerical simulation and mechanistic insight, J. Fluid Mech. 737, R4 (2013).

[22] J. K. G. Dhont and W. J. Briels, Gradient and vorticity banding, Rheol. Acta 47, 257 (2008).

[23] A. M. Philippe, C. Baravian, M. Jenny, F. Meneau, and L. J. Michot, Taylor-Couette Instability in Anisotropic Clay Suspensions Measured Using Small-Angle X-Ray Scattering, Phys. Rev. Lett. 108, 254501 (2012).

[24] C. Baravian, D. Vantelon, and F. Thomas, Rheological determination of interaction potential energy for aqueous clay suspensions, Langmuir 19, 8109 (2003).

[25] F. Del Giudice, B. V. Cunning, R. S. Ruoff, and A. Q. Shen, Filling the gap between transient and steady shear rheology of aqueous graphene oxide dispersions, Rheol. Acta 57, 293 (2018).

[26] G. S. Beavers and D. D. Joseph, Tall Taylor cells in polyacrylamide solutions, Phys. Fluids 17, 650 (1974).

[27] C. S. Dutcher and S. J. Muller, Spatio-temporal mode dynamics and higher order transitions in high aspect ratio Newtonian Taylor-Couette Flows, J. Fluid Mech. 641, 85 (2009).

[28] R. C. DiPrima, P. M. Eagles, and B. S. Ng, The effect of radius ratio on the stability of Couette flow and Taylor vortex flow, Phys. Fluids 27, 2403 (1984).

[29] H. Brenner, Rheology of a dilute suspension of axisymmetric Brownian particles, Int. J. Multiphase Flow 1, 195 (1974).

[30] F. Folgar and C. L. Tucker III, Orientation behavior of fibers in concentrated suspensions, J. Reinf. Plast. Compos. 3, 98 (1984).

[31] E. S. G. Shaqfeh and G. H. Fredrickson, The hydrodynamic stress in a suspension of rods, Phys. Fluids 2 , 7 (1990).

[32] D. L. Koch, A model for orientational diffusion in fiber suspensions, Phys. Fluids 7, 2086 (1995).

[33] C. A. Stover, D. L. Koch, and C. Cohen, Observations of fibre orientation in simple shear flow of semidilute suspensions, J. Fluid Mech. 238, 277 (1992).

[34] M. Rahnama, D. L. Koch, and E. S. G. Shaqfeh, The effect of hydrodynamic interactions on the orientation distribution in a fiber suspension subject to simple shear flow, Phys. Fluids 7, 487 (1995).

[35] A. B. D. Brown, S. M. Clarke, P. Convert, and A. R. Rennie, Orientational order in concentrated dispersions of plate-like kaolinite particles under shear, J. Rheol. 44, 221 (2000).

[36] T. S. Tran, S. J. Park, S. S. Yoo, T.-R. Lee, and T. Y. Kim, High shear-induced exfoliation of graphite into high quality graphene by Taylor-Couette flow, RSC Adv. 6, 12003 (2016). 